\title{
Deterministic pilot pattern allocation optimization for sparse channel estimation based on CS theory in OFDM system
}

\author{
Yang $\mathrm{Nie}^{1,2^{*}}$, Xinle $\mathrm{Yu}^{1}$ and Zhanxin Yang ${ }^{1}$
}

\begin{abstract}
Compressed sensing (CS)-based sparse channel estimation requires the sensing matrix with the minimum mutual coherence $(\mathrm{MC})$, and its corresponding pilot pattern obtain optimal estimation performance. In order to minimize the $M C$ of the sensing matrix, a deterministic optimized pilot pattern allocation scheme based on modified adaptive genetic algorithm (MAGA) is investigated in this paper. By adjusting the probability of mutation and crossover adaptively, the proposed scheme guides the search process to obtain the optimized pilot pattern. This method guarantees the convergence of the optimization process and prevents the process into local optimization to get the global optimization. Compared with the existing methods, simulation results prove that the proposed scheme obtain the sensing matrix with the smaller MC, whose corresponding deterministic pilot pattern effectively improve channel estimation performance.
\end{abstract}

Keywords: Sparse channel estimation, Pilot pattern, Mutual coherence, Compressed sensing

\section{Introduction}

Orthogonal frequency division multiplexing (OFDM) is utilized to resist multipath fading for good performance in wireless communication systems. The coherent demodulation and channel equalization of the receiver all need precise channel state information (CSI) and therefore the channel estimation plays a crucial role. In recent years, compressed sensing (CS) has become an innovative signal processing and acquiring theory by solving optimization problems [1-3]. Comparing the traditional scheme, CS-based sparse channel estimation gets the utmost out of the inherent sparse characteristics of the channel to perform the channel estimation. This method can obtain accurate CSI with much fewer pilots, and increase the spectrum utilization while improving the channel estimation performance [4].

To channel estimation scheme based on CS, the channel impulse response (CIS) is reconstructed by the orthogonal matching pursuit (OMP) algorithm, when

\footnotetext{
*Correspondence: nieyangwork@163.com
'Engineering Research Centre of Digital Audio and Video Ministry of

* Correspondence: nieyangwork@163.com
'Engineering Research Centre of Digital Audio and Video Ministry of Education, Communication University of China, Beijing, China

${ }^{2}$ Key Laboratory of High Speed Signal Processing and Internet of Things

Technology Application, Jining Normal University, Jining, Inner Mongolia, China
}

pilots are randomly distributed in the subcarriers of the OFDM system [5]. If the position of the pilot pattern is randomly selected, the corresponding sensing matrix is a random structure and the restricted isometry property (RIP) is easy to be satisfied. If the pilot pattern location is fixed, the sensing matrix is deterministic and needs to be carefully designed to satisfy RIP. However, a direct and effective method to determine whether the sensing matrix satisfies RIP has not been proposed, and computing the mutual coherence (MC) of the sensing matrix is a good alternative [6]. We utilize the minimum $\mathrm{MC}$ as the criterion for optimizing the allocation of pilot patterns to perform channel estimation based on CS. However, it is unrealistic to blindly search the optimal pilot pattern in the actual communication system. Therefore, it is meaningful to establish an effective pilot pattern allocation optimization method.

Pilot pattern optimization allocation methods have been presented respectively to get the optimal pilot pattern for sparse channel estimation [7-9]. However, these methods are essentially random search. To overcome the disadvantages of random search, genetic algorithm (GA) was introduced to optimize the pilot pattern by updating the individual to find the suboptimal pilot 
pattern [10]. Nevertheless, this method requires the probability of mutation and crossover of being continuously verified to find a suitable value, and it is extremely difficult to select the best value for various optimization problems.

In this paper, modified adaptive genetic algorithm (MAGA)-based pilot pattern allocation optimization scheme is proposed for OFDM sparse channel estimation based on CS Theory. In MAGA, the probability of mutation and crossover is adjusted adaptively by individual fitness. If the solution set has a tendency to trap in local optimal, the probability of mutation and crossover is improved adaptively. If the solution set is scattered in the solution space, the probability of mutation and crossover is reduced adaptively. Therefore, the pilot pattern optimization scheme based on MAGA can greatly guarantee the convergence accuracy of the pilot pattern search process, and effectively avoid obtaining the local optimal solution.

The remainder of this paper is structured as follows. We establish the CS-based channel estimation model, and transform it into an optimization problem in Section 2. The innovative contribution of this work is detailed in Section 3, where MAGA-based pilot pattern allocation optimization scheme is presented and applied to solving the optimization problem. In Section 4, the numerical results of computer simulation are performed to verify the performance of the proposed scheme. Finally, Section 5 generalizes the main conclusion of this paper.

\section{CS-based channel estimation optimization problem}

It is assume that the number of subcarriers is $N$ and the subcarriers $N_{P}$ are chosen as transmitting pilot in OFDM systems. The allocation position of the pilot pattern is $K=\left(k_{1}, \cdots, k_{N_{p}}\right) \quad\left(1 \leq k_{1} \leq \cdots \leq k_{N_{p}} \leq N\right)$. The relationship between the received pilot and transmitted pilot is expressed as

$$
Y_{N_{p}}=X_{N_{p}} F_{N_{p}} h+W_{N_{p}}
$$

where a diagonal matrix $X_{N_{P}}=\operatorname{diag}\left[X\left(k_{1}\right), X\left(k_{2}\right), \cdots, X\right.$ $\left.\left(k_{N_{P}}\right)\right]$ is composed of the transmitted pilot, the received pilot is $Y_{N_{P}}=\left[Y\left(k_{1}\right), Y\left(k_{2}\right), \cdots, Y\left(k_{N_{P}}\right)\right]^{T}$, and the channel impulse response $h=[h(1), h(2), \cdots, h(N)]^{T}$ is of the length of $N$, among which the first $L$ elements contain multipath energy. $W_{N_{P}}=\left[W\left(k_{1}\right), W\left(k_{2}\right), \cdots, W\left(k_{N_{P}}\right)\right]^{T}$ is the Gaussian white noise in the frequency domain. $F_{N_{P}}$ is a $N_{P} \times N$ partial Fourier matrix, whose $N_{P}$ row is extracted from the $N \times N$ standard Fourier matrix by the pilot pattern $K$, and defined as

$$
F_{N_{p}}=\frac{1}{\sqrt{N}}\left[\begin{array}{ccc}
f^{k_{1} 1} & \ldots & f^{k_{1} N} \\
\vdots & \ddots & \vdots \\
f^{k_{N_{p}} 1} & \ldots & f^{k_{N_{p}} N}
\end{array}\right],
$$

where $f=e^{-j 2 \pi / N}$. We further denote

$$
A \cong X_{N_{p}} F_{N_{p}}=\left[\begin{array}{ccc}
X\left(k_{1}\right) f^{k_{1} 1} & \cdots & X\left(k_{1}\right) f^{k_{1} N} \\
\vdots & \ddots & \vdots \\
X\left(k_{N_{p}}\right) f^{k_{N_{p}} 1} & \cdots & X\left(k_{N_{p}}\right) f^{k_{N_{p}} N}
\end{array}\right] .
$$

Then, (1) can be rewritten as

$$
Y_{N_{p}}=A h+W_{N_{p}} .
$$

Since the sampled interval is usually much smaller than the channel delay propagation, the channel coefficients are either zero or nearly zero, which implies that $h$ is a sparse vector. According to the CS theory, the matrix $A$ can be regarded as the sensing matrix, and it is essentially the weight of the transmitted pilot signal to the partial Fourier matrix. If the placement of the pilot pattern is randomly selected, the matrix $A$ is a structured random matrix weighted by the transmitted pilot. Correspondingly, if the placement of the pilot pattern is deterministic, it is a deterministic sensing matrix. Therefore, the process of channel estimation based on CS is explained that the transmitted pilot is compressed to measure the impulse response $h$, and then $h$ is reconstructed by the reconstruction algorithm with the received pilots. Moreover, it can clearly be seen from (3) that the pilot pattern decides the extraction of those rows of the standard Fourier transform matrix, and then determines the structure of the sensing matrix, and ultimately affects the performance of OFDM channel estimation. If the allocation of the pilot pattern is random, the corresponding sensing matrix is the structured random matrix. However, the pilot subcarrier with random distribution is not easy to be realized in the actual communication system. Therefore, it is necessary to study the deterministic sensing matrix with optimizing pilot pattern allocation so as to ensure the channel estimation performance.

When the sensing matrix satisfies the RIP, the channel impulse response can be recovered from the received pilot with a high probability. However, there is no known method to test whether a given matrix satisfies RIP in polynomial time. Alternatively, we can compute the $\mathrm{MC}$ of the sensing matrix $A$ to verify RIP and it can be formulated as

$$
\mu(A)=\max _{1 \leq m \leq N \leq N} \frac{\left|\left\langle a_{m}, a_{n}\right\rangle\right|}{\left\|a_{m}\right\|_{2}\left\|a_{n}\right\|_{2}},
$$

where $\left|\left\langle a_{m}, a_{n}\right\rangle\right|$ denotes the inner product between the $m$ th column and the $n$th column of the sensing matrix $A$. Substituting (3) to (5) can be obtained 


$$
\begin{aligned}
\mu(A) & =\max _{1 \leq m_{m \neq n} n \leq N} \frac{\frac{1}{N}\left|\sum_{i=1}^{N_{p}} \overline{X\left(k_{i}\right)} e^{j 2 \pi k_{i} m / N} X\left(k_{i}\right) e^{-j 2 \pi k_{i} n / N}\right|}{\frac{1}{\sqrt{N}}\left(\sum_{i=1}^{N_{p}}\left|X\left(k_{i}\right) e^{-j 2 \pi k_{i} m / N}\right|^{2}\right)^{\frac{1}{2}} \frac{1}{\sqrt{N}}\left(\sum_{i=1}^{N_{p}}\left|X\left(k_{i}\right) e^{-j 2 \pi k_{i} n / N}\right|^{2}\right)^{\frac{1}{2}}} \\
& =\max _{1 \leq m<n \leq N} \frac{\left.\left|\sum_{i=1}^{N_{p}}\right| X\left(k_{i}\right)\right|^{2} e^{-j 2 \pi k_{i}(n-m) / N} \mid}{\sum_{i=1}^{N_{p}}\left|X\left(k_{i}\right)\right|^{2}} .
\end{aligned}
$$

Since the pilots used in the OFDM system satisfy the constant amplitude, we define $\left|X\left(k_{i}\right)\right|=1 \quad\left(i=1,2 \cdots N_{p}\right)$. Let $d=n-m$, and $\Omega=\{1,2, \cdots N-1\}$. Then (6) can be rewritten as

$$
\mu(A)=\max _{d \in \Omega} \frac{1}{N_{p}}\left|\sum_{i=1}^{N_{p}} e^{-j 2 \pi k_{i} d / N}\right| .
$$

It can be seen from (7) that the MC of matrix $A$ is determined by the pilot pattern $K$, when the number of subcarrier and pilot has been determined.

As mentioned in Section 1, the pilot pattern is optimized by the rule of minimizing the MC of the sensing matrix, and the sparse channel impulse response $h$ is reconstructed based on CS algorithm. Replacing the $A$ in (7) with $K$ and taking it as a variable, the optimized position problem of the pilot pattern allocation can be expressed as

$$
Q=\min _{K \in P} \mu(K) .
$$

where $P$ is the set of all possible pilot patterns. In the OFDM communication system, it is unrealistic to search all pilot patterns to determine the optimal pilot pattern because the number of pilot pattern is huge and the computational complexity is very high. Therefore, it is necessary to propose an optimization method to solve Eq. (8) for reducing computational complexity and obtaining optimal pilot pattern.

\section{Method: MAGA-based pilot pattern optimization}

In Section 2, the pilot pattern allocation optimization is transformed into a combinatorial optimization problem, and the optimal pilot pattern is

$$
K_{\text {opt }}=\underset{K \in P}{\arg \min } \mu(K) .
$$

If the enumeration method is selected for searching the optimal pilot pattern, the computation complexity is huge. Therefore, a new pilot pattern optimization method is of great necessity. This method can quickly solve the combinatorial optimization problem (8) by adaptive adjustment of genetic operators, and then the optimized pilot pattern is acquired for efficient detection of sparse channels.

\subsection{MAGA}

Genetic algorithm (GA) is an intelligent optimization algorithm inspired by natural evolution. This algorithm applies the selection, mutation, and crossover to obtain the new population, which gradually evolves to get the optimal solution. Mutation probability and crossover
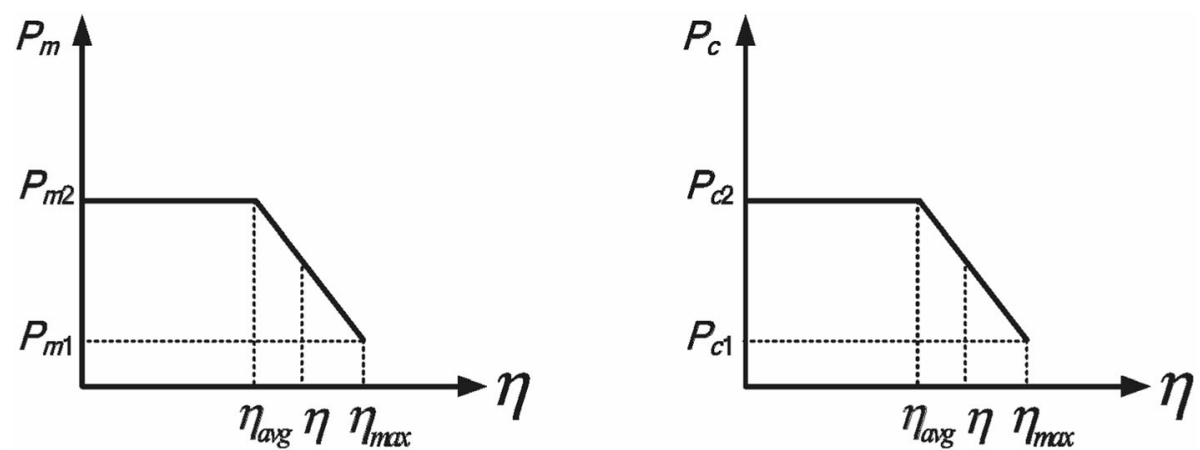

Fig. 1 MAGA-based mutation probability and crossover probability. The distribution of mutation probability and cross probability based on MAGA with individual fitness is displayed 


\section{Algorithm: Modified Adaptive Genetic Algorithm Pilot pattern optimization \\ 1: Initialization. The iteration number $\mathrm{T}$ is initialized, and the maximum number of evolution $\mathrm{M}$ is assigned. The population generates $\mathrm{N}$ random pilot patterns, which are used as the starting point for population evolution. \\ 2: The fitness of individuals in the population is calculated and sorted according to value to prepare for the next genetic operation. The minimum mutual coherence of sensing matrix is used to evaluate the fitness of individuals in MAGA. \\ 3: Selection.According to individual fitness, some excellent individuals are selected to inherit the next generation population by roulette rule. \\ 4: Crossover. According to formula (16), the crossover probability is obtained and some chromosomes in the population are exchanged to combine the genetic information of two parents to generate new offspring. \\ 5: Mutation. According to formula (17), the mutation probability is obtained and the gene values of the selected individuals are changed to generate new individuals. \\ 6: Judgement of termination condition: if it is not satisfied, go to step 2; if it is satisfied, stop optimizing. \\ Output: optimal pilot pattern, which is the individual with the greatest fitness in the iterative process.}

Fig. 2 MAGA-based pilot pattern allocation optimization scheme. The deterministic pilot pattern optimization algorithm based on MAGA is described in detail, which includes initialization, selection, crossover, mutation, and optimal pilot output

probability largely determine the accuracy and convergence speed in the optimization process. If the value of the crossover probability is larger, the production rate of new individuals in the population will be accelerated, and the destruction possibility of individuals with high fitness will be increased. If the crossover probability is smaller, the search time of the algorithm will become longer or even pause. Furthermore, if the mutation probability is larger, the algorithm becomes random search. If the value is smaller, the new population is not easy to generate. Therefore, how to determine mutation probability and crossover probability is very important in the GA. On the other hand, mutation probability and crossover probability often need to be repeatedly verified by manual experience. Therefore, it is hard to select the appropriate value for different optimization problems.

Adaptive genetic algorithm (AGA) was proposed in order to perfect genetic algorithm [11]. In the AGA, mutation probability and crossover probability are adaptively obtained by the individual fitness. If the solution has the local optimal tendency, mutation probability and crossover probability will be increased adaptively. For excellent individuals, we should reduce the probability of crossover and the probability of mutation to protect them. For inferior individuals, we should increase the probability of crossover and the probability of mutation to change them. Therefore, for the optimization problem based on adaptive genetic algorithm, mutation probability, and crossover probability are obtained adaptively, which effectively ensures the convergence of the optimization and enlarges the population diversity. However, the AGA is suitable for later evolution of population. This is because the individual performance of the population is excellent, so we should protect the chromosomal structure from being destroyed [12]. In the early stage of evolution, the individuals with good performance will

Table 1 System-related parameters

\begin{tabular}{ll}
\hline Parameters & Value \\
\hline OFDM sample period & $T=83.3 \mu \mathrm{s}$ \\
OFDM symbol period & $T_{\mathrm{u}}=21.33 \mathrm{~ms}$ \\
Guard interval & $T_{\mathrm{g}}=5.3 \mathrm{~ms}$ \\
FFT length & $\mathrm{N}=256$ \\
Number of channel multipath & $\mathrm{S}=4$ \\
Modulation & $4 \mathrm{QAM}$ \\
Number of pilot & $\mathrm{M}=26$ \\
SNR & $0-30 \mathrm{~dB}$ \\
\hline
\end{tabular}


Table 2 Optimized pilot patterns by MAGA, GA, and random

\begin{tabular}{|c|c|c|c|}
\hline Type & $M C$ & Running time (s) & Optimized pilot pattern \\
\hline Random & 0.2045 & 570.65 & $\begin{array}{l}4,9,20,25,34,38,65,70,75,83,91,104,125,130,135,149, \\
171,178,187,188,194,202,211,219,226,248\end{array}$ \\
\hline GA & 0.1812 & 140.53 & $\begin{array}{l}20,26,42,53,56,64,72,77,79,85,101,107,114,119,128 \\
146,151,156,160,173,197,208,216,223,228,244\end{array}$ \\
\hline MAGA & 0.1399 & 310.41 & $\begin{array}{l}4,16,31,39,46,54,61,75,92,100,107,113,135,142,160 \\
168,176,183,191,197,205,211,221,229,246,253\end{array}$ \\
\hline
\end{tabular}

hardly change. This will not be conducive to the evolution process, and will easily cause the solution to trap in local optimum [13].

Since the traditional AGA is easy to be trapped into a local-optimal solution, MAGA is presented in an effort to determine a globally optimal solution in this paper. Mutation probability and crossover probability formulas are respectively defined as

$$
P_{c}=\left\{\begin{array}{ll}
P_{c 2}-\frac{P_{c 2}-P_{c 1}}{\eta_{\text {max }}-\eta_{\text {avg }}}\left(\eta-\eta_{\text {avg }}\right) & \eta \geq \eta_{\text {avg }} \\
P_{c 2} & \eta<\eta_{\text {avg }}
\end{array},\right.
$$

$$
P_{m}=\left\{\begin{array}{ll}
P_{m 2}-\frac{P_{m 2}-P_{m 1}}{\eta_{\text {max }}-\eta_{\text {avg }}}\left(\eta-\eta_{\text {avg }}\right) & \eta \geq \eta_{\text {avg }} \\
P_{m 2} & \eta<\eta_{\text {avg }}
\end{array},\right.
$$

where $\eta_{\text {avg }}$ is the population average fitness, $\eta_{\max }$ is the population maximum fitness, and $\eta$ is the individual fitness. The crossover probability minimum value is $P_{c 1}=0.1$, and the maximum value is $P_{c 2}=0.9$. The mutation probability minimum value is $P_{m 1}=0.01$, and the maximum value is $P_{m 2}=0.1$.

Figure 1 illustrates the distribution of values of crossover probability and mutation probability in the

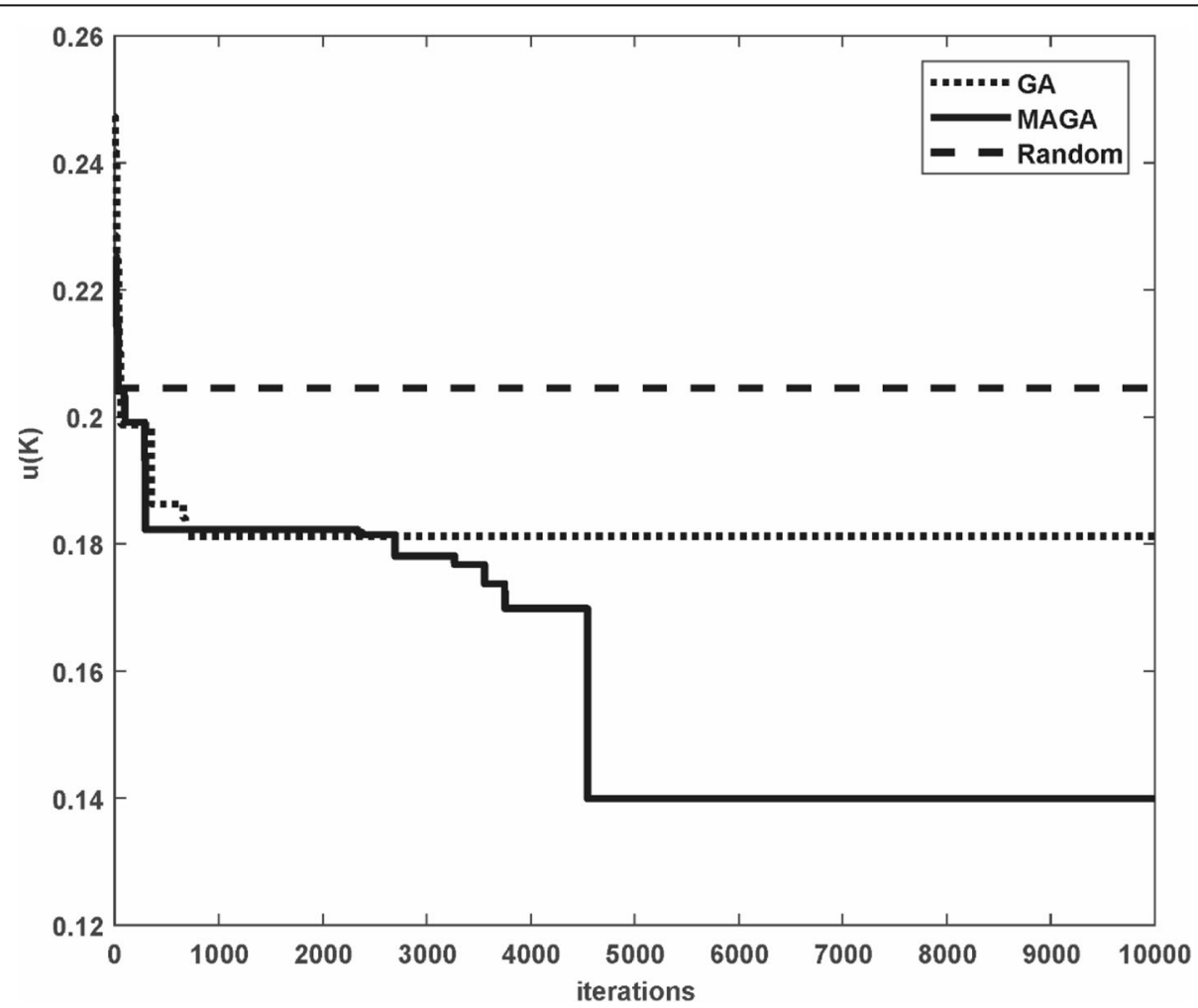

Fig. 3 Comparisons of the MC for different optimized schemes. The MC is optimized by three different optimization algorithms with random search, GA, and MAGA 
MAGA. The mutation probability $P_{m}$ and the crossover probability $P_{c}$ of the largest fitness individuals are not zero in the MAGA, and these values are increased to $P_{c 1}$ and $P_{m 1}$ respectively. The modified algorithm ensures that the optimization process will not stop in the early stage of evolution, which can avoid solutions falling into local optimum.

\subsection{MAGA-based pilot pattern optimization method}

If we select $N_{P}$ subcarriers from the $N$ subcarriers as pilots, then possible pilot patterns will be $\left(\begin{array}{l}N \\ N_{P}\end{array}\right)$. It is very difficult to obtain all the pilot patterns for selecting the optimal. Here, we present a method to globally search for a near-optimal deterministic pilot pattern allocation by MAGA. This scheme is universal for OFDM system with getting the optimized sensing matrix by minimizing the $\mathrm{MC}$, and the specific execution is demonstrated in Fig. 2.

The scheme with the initial population $P^{(0)}$, which consists of randomly obtained $N$ individuals, starts the execution of the work. The $i$ th generation of the evolutionary population is expressed as

$$
P^{(i)}=\left[p_{1}^{(i)}, \ldots, p_{N}^{(i)}\right] \quad(1 \leq i \leq M)
$$

Secondly, according to individual fitness, the roulette rule is used to select some excellent individuals from the $P^{(i)}$ generation to the $P^{(i+1)}$ generation. The probability that an individual of the population will be selected for the next generation is defined as

$$
w_{j}^{i}=\frac{\vartheta_{j}^{i}}{\sum_{j=1}^{N} \vartheta_{j}^{i}} \quad(j=1,2, \cdots, N) .
$$

Then, the genetic operators are adaptive to complete mutation and crossover to get the new population based on the MAGA. The whole optimization process is repeatedly iterated until the stop condition is satisfied. Finally, the individual with the greatest fitness is selected for the output of the optimization process, which is the suboptimal deterministic pilot pattern.

\section{Results and discussion}

Comparing the superiority of our proposed method with the existing scheme, we designed simulation experiments.

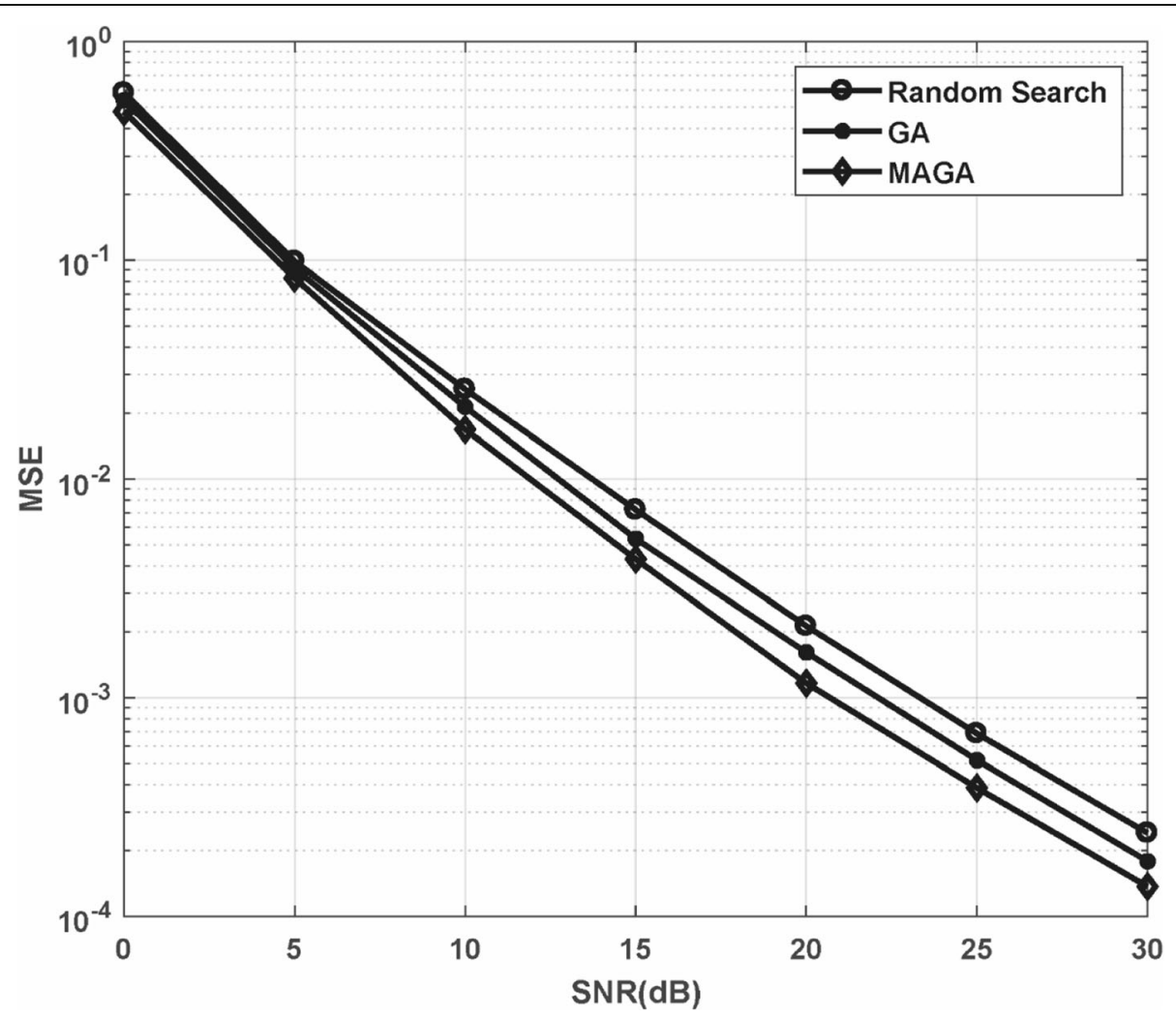

Fig. 4 MSE performance comparisons with different pilot patterns optimized. The performance gain of channel estimation based on CS theory is compared with three different deterministic pilot patterns, which are obtained by random search, GA, and MAGA respectively 
Random search, GA search, and the proposed method are used to find the optimal deterministic pilot pattern. Simulations were accomplished in MATLAB R2017a by an Intel Core i5-3320M $2.60 \mathrm{GHz}$ processor with $8 \mathrm{~GB}$ memory in this section. Simulation experiments were carried out under ideal synchronization and without channel coding. The OMP algorithm is chosen to complete the sparse channel reconstruction. It can achieve good channel estimation performance with fewer pilots. However, the complexity of the algorithm is increased. The complexity of the OMP algorithm is $o\left(L D N_{P}\right)$, where $L$ is the channel length, $D$ is the number of iterations, and $N_{P}$ is the number of pilots. The channel model is channel 3 of mode B in the DRM standard [14], and the related parameters are displayed in Table 1.

Table 2 compares pilot patterns obtained by random search, GA, and MAGA. Optimized pilot patterns are placed in the last column. It is observed that these methods generate different suboptimal pilot patterns. As can be seen in Table 2, the mutual coherence of the pilot pattern optimized based on the MAGA is the smallest. The $\mathrm{MC}$ reduces from 0.2045 to 0.1399 , which verifies the effectiveness of our scheme. The complexity of the pilot pattern generation is compared according to the CPU running time using MATLAB. We observe that the MAGA scheme is more time-consuming than the GA method in Table 2. However, the pilot pattern generation is done offline before the OFDM symbols are transmitted, so the proposed scheme is still acceptable.

Figure 3 shows the optimization process of mutual coherence with different pilot pattern optimization methods. The number of iteration for different schemes is 10,000 . As can be seen from Fig. 3, the proposed method can obtain smaller MC compared with other methods. As demonstrated in Figs. 4 and 5, we compare the channel estimation performance using different optimized pilot patterns to detect sparse channel. The mean square error (MSE) of channel estimation by various optimized pilot pattern schemes is compared in Fig. 4. MAGA outperforms GA and the random search obtained pilots, which verify the practicality of the proposed method. The simulation results show that the smaller $\mathrm{MC}$ of the sensing matrix, the better the channel estimation performance. The system performance in bit error rate (BER) can be displayed in Fig. 5. We have noticed that the pilot generated by MAGA performs best than other optimization methods. Therefore, MAGA-based pilot pattern optimization is a very effective way to enhance the estimation performance based on CS.

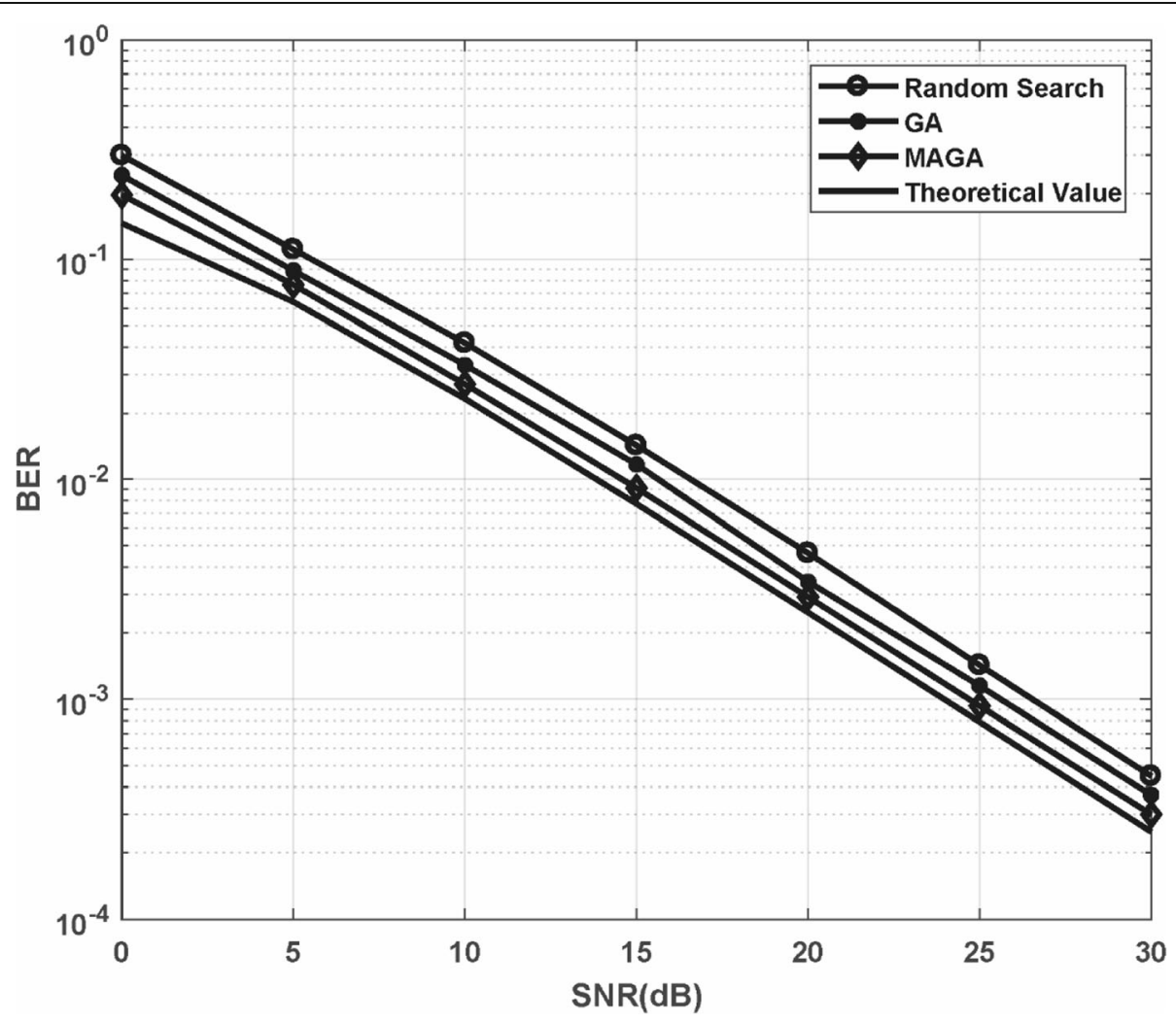

Fig. 5 BER performance comparisons with different pilot patterns optimized. The BER performance of channel estimation based on CS theory is compared with three different deterministic pilot patterns, which are obtained by random search, GA, and MAGA respectively 


\section{Conclusion}

CS is an innovative theory for efficiently processing and acquiring data by solving an underdetermined linear equation. CS-based sparse channel estimation can reconstruct channel impulse response with less pilot signals to solve the optimization problem, which are far fewer than those required by the sampling theory. This method has shown the advantages of increasing the estimation performance and reducing the pilot number by taking advantage of the inherent sparse characteristics of wireless channels. According to the CS theory, the smaller MC of the sensing matrix is beneficial to enhance estimation performance. An optimal scheme of deterministic pilot pattern allocation based on MAGA is proposed in this paper, which aims at minimizing the MC of the sensing matrix to pilot pattern. The proposed scheme can adaptively acquire the mutation probability and the crossover probability, and guide the optimization process to generate a near-optimal deterministic pilot pattern. The results of computer simulation prove that the proposed method is able to obtain a smaller MC sensing matrix compared with the existing schemes, and the estimation performance with the optimized pilot pattern is substantially improved for the OFDM sparse channel estimation based on CS theory.

\begin{abstract}
Abbreviations
AGA: Adaptive genetic algorithm; BER: Bit error rate; CS: Compressed sensing; CSI: Channel state information; GA: Genetic algorithm; MAGA: Modified adaptive genetic algorithm; MC: Mutual coherence; MSE: Mean square error; OFDM: Orthogonal frequency division multiplexing; OMP: Orthogonal matching pursuit; RIP: Restricted isometry property
\end{abstract}

\section{Acknowledgements}

Engineering Research Centre of Digital Audio and Video Ministry of Education, Communication University of China and Key Laboratory of High Speed Signal Processing and Internet of Things Technology Application, Jining Normal University.

\section{Funding}

This work was supported by Fundamental Research Funds for the Central Universities (Grant: 3132017XNG17), National Key Technology Research and Development Program of the Ministry of Science and Technology of China (Grant:2015BAK05B01),Research Program of Science and Technology at Universities of Inner Mongolia Autonomous Region (Grant: NJZY18232), and Research Fund for the Doctoral Program of Jining Normal University (Grant: jsbsjj1801).

\section{Availability of data and materials}

Not applicable.

\section{Authors' contributions}

YN is the main contributor and author of this paper. He presented the main idea, formulated the model of channel estimation, accomplished simulation experiment, and analyzed the experimental result. XY completed the OMP algorithm. ZY proposed some meaningful suggestions for sparse channel estimation. All authors read and approved the final manuscript.

\section{Publisher's Note}

Springer Nature remains neutral with regard to jurisdictional claims in published maps and institutional affiliations.

Received: 29 June 2018 Accepted: 21 December 2018

Published online: 08 January 2019

\section{References}

1. D.L. Donoho, Compressed sensing. IEEE Trans. Inf. Theory 52(4), 1289-1306 (2006)

2. R.G. Baraniuk, Compressive sensing. IEEE Signal Process. Mag. 24(4), 118-121 (2007)

3. E.J. Candès, M.B. Wakin, An introduction to compressive sampling. IEEE Signal Process. Mag. 25(2), 21-30 (2008)

4. W.U. Bajwa, J. Haupt, A.M. Sayeed, et al., Compressed channel sensing: a new approach to estimating sparse multipath channels. Proc. IEEE 98(6), 1058-1076 (2010)

5. C.R. Berger, S. Zhou, J.C. Preisig, et al., Sparse channel estimation for multicarrier underwater acoustic communication: from subspace methods to compressed sensing. IEEE Trans. Signal Process. 58(3), 1708-1721 (2010)

6. J.A. Tropp, Greed is good: algorithmic results for sparse approximation. IEEE Trans. Inf. Theory 50(10), 2231-2242 (2004)

7. X. He, R. Song, W.P. Zhu, Optimal pilot pattern design for compressed sensing-based sparse channel estimation in OFDM systems. Circuits Syst. Signal Proc. 31(4), 1379-1395 (2012)

8. C. Qi, L. Wu, Optimized pilot placement for sparse channel estimation in OFDM systems. IEEE Signal Proc Lett. 18(12), 749-752 (2011)

9. C. Qi, L. Wu, A study of deterministic pilot allocation for sparse channel estimation in OFDM systems. IEEE Commun. Lett. 16(5), 742-744 (2012)

10. X. He, R. Song, W.P. Zhu, Pilot allocation for sparse channel estimation in MIMOOFDM systems. IEEE Trans. Circuits Syst. Express Briefs 60(9), 612-616 (2013)

11. M. Srinivas, L.M. Patnaik, Adaptive probabilities of crossover and mutation in genetic algorithms. IEEE Trans. Syst. Man Cybern. 24(4), 656-667 (1994)

12. K. Deb, H.G. Beyer, Self-adaptive genetic algorithms with simulated binary crossover. Evol. Comput. 9(2), 197-221 (2001)

13. Z. Ye, Z. Li, M. Xie, Some improvements on adaptive genetic algorithms for reliability-related applications. Reliab. Eng. Syst. Saf. 95(2), 120-126 (2010)

14. C. Qi, L. Wu, Tree-based backward pilot generation for sparse channel estimation. Electron. Lett. 48(9), 501-503 (2012)

\section{Submit your manuscript to a SpringerOpen ${ }^{\mathcal{O}}$ journal and benefit from:}

- Convenient online submission

- Rigorous peer review

- Open access: articles freely available online

High visibility within the field

- Retaining the copyright to your article

Submit your next manuscript at $\boldsymbol{\nabla}$ springeropen.com 